\title{
Schistosoma mansoni
}

National Cancer Institute

\section{Source}

National Cancer Institute. Schistosoma mansoni. NCI Thesaurus. Code C124391.

A species of flatworms in the family Schistosomatidae. The lifecycle of S. mansoni involves Biomphalaria snails as the intermediate host and humans as the definitive host.

Infestation is most often found in the superior mesenteric veins draining the large intestine. This schistosome is a causative agent of intestinal schistosomiasis. 